\title{
Autoimmun-Retinopathie im Zusammenhang mit monoklonaler Gammopathie unklarer Signifikanz: ein Fallbericht
}

\author{
Emily A. Eton ${ }^{a} \quad$ Gary Abrams $^{\mathrm{b}} \quad$ Naheed W. Kahn $^{\mathrm{a}} \quad$ Abigail T. Fahim $^{\mathrm{a}}$ \\ ${ }^{a}$ Kellogg Eye Center, University of Michigan, Ann Arbor, MI, USA; ${ }^{b}$ Kresge Eye Institute, Wayne State University, Detroit, MI, USA
}

\section{Schlüsselwörter}

Autoimmun-Retinopathie · Monoklonale Gammopathie unklarer Signifikanz · Plasmazelldyskrasien

\section{Abstract}

Hintergrund: Monoklonale Gammopathie unklarer Signifikanz (MGUS) ist eine Plasmazelldyskrasie und Vorstufe des Multiplen Myeloms. Okuläre Manifestationen sind bekannt, bislang wurde jedoch noch kein Zusammenhang mit einer Autoimmun-Retinopathie nachgewiesen.

Fallvorstellung: Eine 57-jährige Frau stellte sich mit seit einem Jahr bestehendem progressiven, beidseitigen, peripheren Verlust des Sehvermögens, Photopsien und Nachtblindheit vor. Die Augenhintergrunduntersuchung und umfassende ergänzende Tests legten den Verdacht einer hereditären oder einer Autoimmun-Retinopathie nahe. Man stellte bei der Patientin antiretinale Antikörper gegen Carboanhydrase II- und Enolase-Proteine bei einem negativen Gen-Panel für Netzhautdystrophie fest. Die Untersuchung auf Malignität war negativ, aber bei der Patientin wurde MGUS, eine prämaligne Erkrankung, diagnostiziert. Die Patientin wurde mit Immunsuppressiva behandelt, wobei sie im Hinblick auf ihre Symptomatik und die augenmedizinischen Untersuchungen am deutlichsten auf Rituximab ansprach.

Schlussfolgerung: MGUS sollte bei Patienten ohne andere autoimmune oder maligne Krankheitsprozesse als potenziell ätiologisch für eine Autoimmun-Retinopathie in Betracht gezogen werden. Eine Behandlung mit Immunsuppressiva kann bei der Begrenzung der Krankheitsprogression von Nutzen sein, und Rituximab erweist sich bei Patienten mit Retinopathie, die auf andere Wirkstoffe nicht ansprechen, als wirksam.

(c) 2020 The Author(s)

\section{Hintergrund}

Bei der monoklonalen Gammopathie unklarer Signifikanz (MGUS) handelt es sich um eine prämaligne Proliferation klonaler Plasmazellen, die durch das Vorliegen von M-Protein im Serum ohne damit verbundene Symptome von Endorganschäden gekennzeichnet ist, wie sie bei Multiplem Myelom auftreten (Hyperkalzämie, Niereninsuffizienz, Anämie, Knochenläsionen) [1]. MGUS ist mit dem Risiko einer Progression zu malignen Erkrankungen verbunden: Bei 11\% der Patienten schreitet MGUS zu Multiplem Myelom, anderen Plasmazellmalignitäten oder Lymphomen fort [2]. Okuläre Manifestationen von MGUS sind selten, wurden jedoch in der Literatur beschrieben und umfassen Maku- lopathie mit seröser Makulaablösung [3], kristalline Keratopathie [4] und Kupferablagerungen in der Descemet-Membran [5]. Wir berichten über einen neuartigen Fall von Autoimmun-Retinopathie bei einer Patientin mit MGUS.

\section{Fallvorstellung}

Eine 57-jährige Frau wurde mit seit einem Jahr bestehendem progressiven peripheren Verlust des Sehvermögens zu uns überwiesen. Weitere Symptome waren u.a. fortschreitende Nachtblindheit seit 6 Monaten und das Auftreten von Photopsien seit 2 Monaten. In der ophthalmologischen Vorgeschichte sind insbesondere sklerotische Kernkatarakte beidseitig und eine vollstän- 
Abb. 1. Goldmann-Gesichtsfeldmessungen (Goldmann Visual Fields, GVF) wurden für die Ziele IV4e (rot), Ill4e (grün) und I4e (schwarz) durchgeführt. Die Gesichtsfelder bei Baseline des LA (a) und RA (b) zeigten eine starke Einengung der Isoptere 14 e und eine mittelgradige Einengung der Isopteren III4e und IV4e mit vergrößerten blinden Flecken und vereinzelten Skotomen in der mittleren Netzhautperipherie beidseitig. Sechs Monate später zeigten sich Verbesserungen bezüglich der Einengung und Skotome im LA (c) und RA (d) unter Prednison und Methotrexat. Fünf Monate nach Beginn der Behandlung mit Methotrexat zeigte sich eine erneute Verschlechterung der Einengung aller Isopteren im LA (e) und RA (f) trotz steigender Methotrexat-Dosierung. Die Einengung der Isopteren IV4e und III4e verbesserte sich im $L A(\mathbf{g})$ und RA (h) nach Beginn der Rituximab-Infusionen.

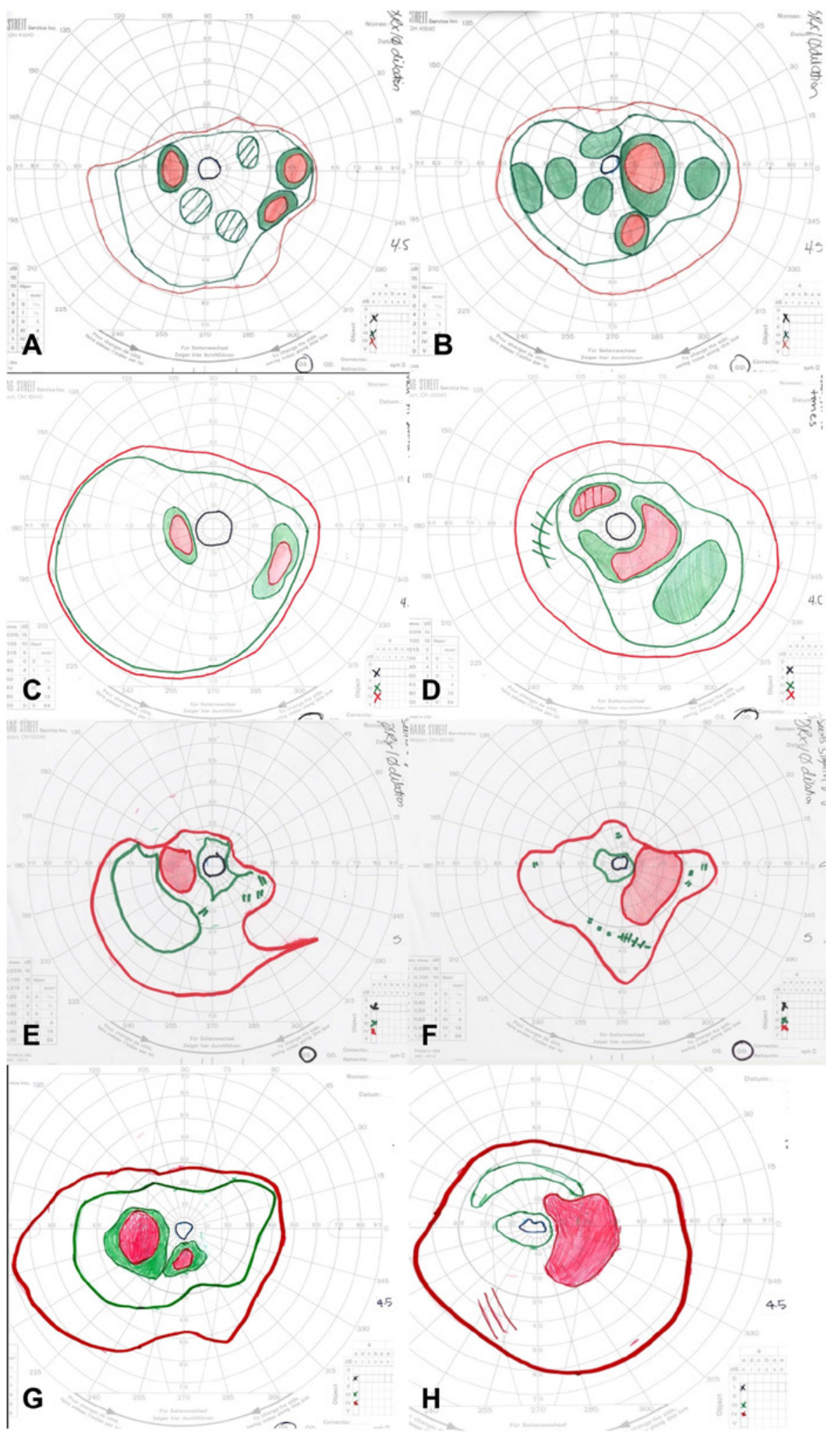

dige hintere Glaskörperabhebung beidseitig anzumerken. Die medizinische Vorgeschichte war signifikant für insulinabhängigen Diabetes mellitus Typ 2 ohne Retinopathie und Hypertonie. Bezüglich der augenmedizinischen Familienanamnese berichtete die Patientin über einen Bruder mit Glaukom.
Die ophthalmologische Untersuchung ergab einen besten korrigierten Visus von 20/25 beidseitig. Eine Untersuchung des Farbsehvermögens mittels Ishihara-Farbtafeln und Farnsworth-Test (D-15) ergab keine Defizite. Die Goldmann-Perimetrie (Goldmann-Gesichtsfeldmessung) zeigte eine mittel- bis hochgradige 
Abb. 2. Ultraweitwinkel-Farbaufnahmen des Augenhintergrunds des RA (a) und LA (b) zeigten eine leichte Blässe der Papille, eine Abschwächung der Netzhautgefäße sowie eine Pigmentierung von Knochenkörperchen in der mittleren Peripherie, im RA stärker als im LA.

Abb. 3. Die OCT der Makula ergab eine erhaltene zentrale Insel der Ellipsoidschicht und der äußeren Kernschicht im RA (a) und LA (b) bei ansonsten schwerer Atrophie der äußeren Retina und Spuren von intraretinaler Zystenflüssigkeit im RA (c). Nach sechsmonatiger Behandlung mit Prednison und Methotrexat hatte sich die intraretinale Flüssigkeit im RA aufgelöst (d).
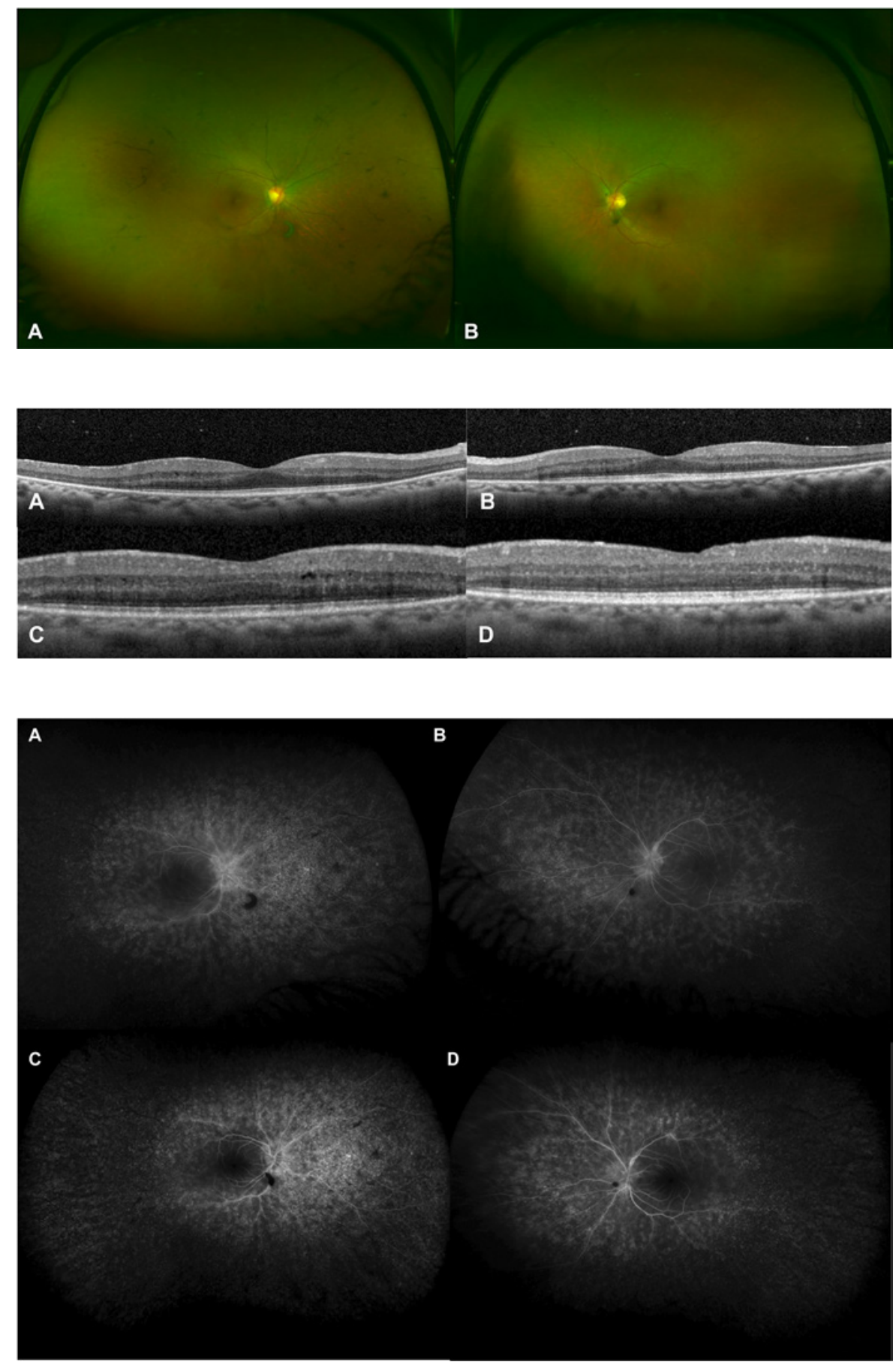

Abb. 4. Eine bei Erstvorstellung der Patientin durchgeführte Fluoreszenzangiographie zeigte in der Spätphase vereinzelte Bereiche tiefer Leckagen mit fehlender peripherer Perfusion und Leckagen im Bereich der Sehnervpapille, im RA (a) stärker als im LA (b). Nach sechsmonatiger Behandlung mit Immunsuppressiva war ein verringertes Zeitintervall für die Leckagen im RA (c) und LA (d) erkennbar. beidseitige Einengung mit vergrößerten blinden Flecken und vereinzelten Skotomen in der mittleren Netzhautperipherie (Abb. 1 a-b). Die mit Hilfe eines Goldmann-Weekers-Adaptometers nach 45 Min. in der Dunkelheit gemessene Dunkeladaptationsschwelle war im rechten Auge (RA) um 1,0 log-Einheiten bei Fixation und 2,5 log-Einheiten peripher und im linken Auge (LA) um 0,7 log-Einheiten bei Fixation und 2,5 log-Einheiten peripher erhöht. Der Augeninnendruck betrug $15 \mathrm{mmHg}$ beidseitig mit direkter Pupillenreaktion ohne afferente Pupillenschädigung. Die Untersuchung des anterioren Segments mittels Spaltlampe ergab insbesondere tiefe und ruhige vordere Augenkammern mit 1+ Kern- sklerose beidseitig sowie Nachweis von Vorderkammerzellen lediglich im RA. Bei der Augenhintergrunduntersuchung zeigten sich Weiss-Ring-Floater beidseitig, eine leichte Blässe der Papille sowie erheblich abgeschwächte Netzhautgefäße mit perivaskulärem Pigment in beiden Augen. In der mittleren Netzhautperipherie fand sich eine mittelstarke Pigmentierung von Knochenkörperchen, die im RA stärker war als im LA. (Abb. 2). Eine optische Kohärenztomografie (OCT) der Makula ergab eine schwere Atrophie der äußeren Retina mit zentraler Aussparung der Ellipsoidzone und äußeren Kernschicht beidseitig sowie Spuren von intraretinaler Zystenflüssigkeit im RA (Abb. 3 a-c). Eine darüber hin- 
Abb. 5. Die Fundusautofluoreszenz ergab eine leicht verstärkte umlaufende Autofluoreszenz im parafovealen Bereich des RA (a) und LA (b) mit Hyper- und Hypoautofluoreszenz-Sprenkeln im RA entlang der superioren und inferioren Gefäßbögen.
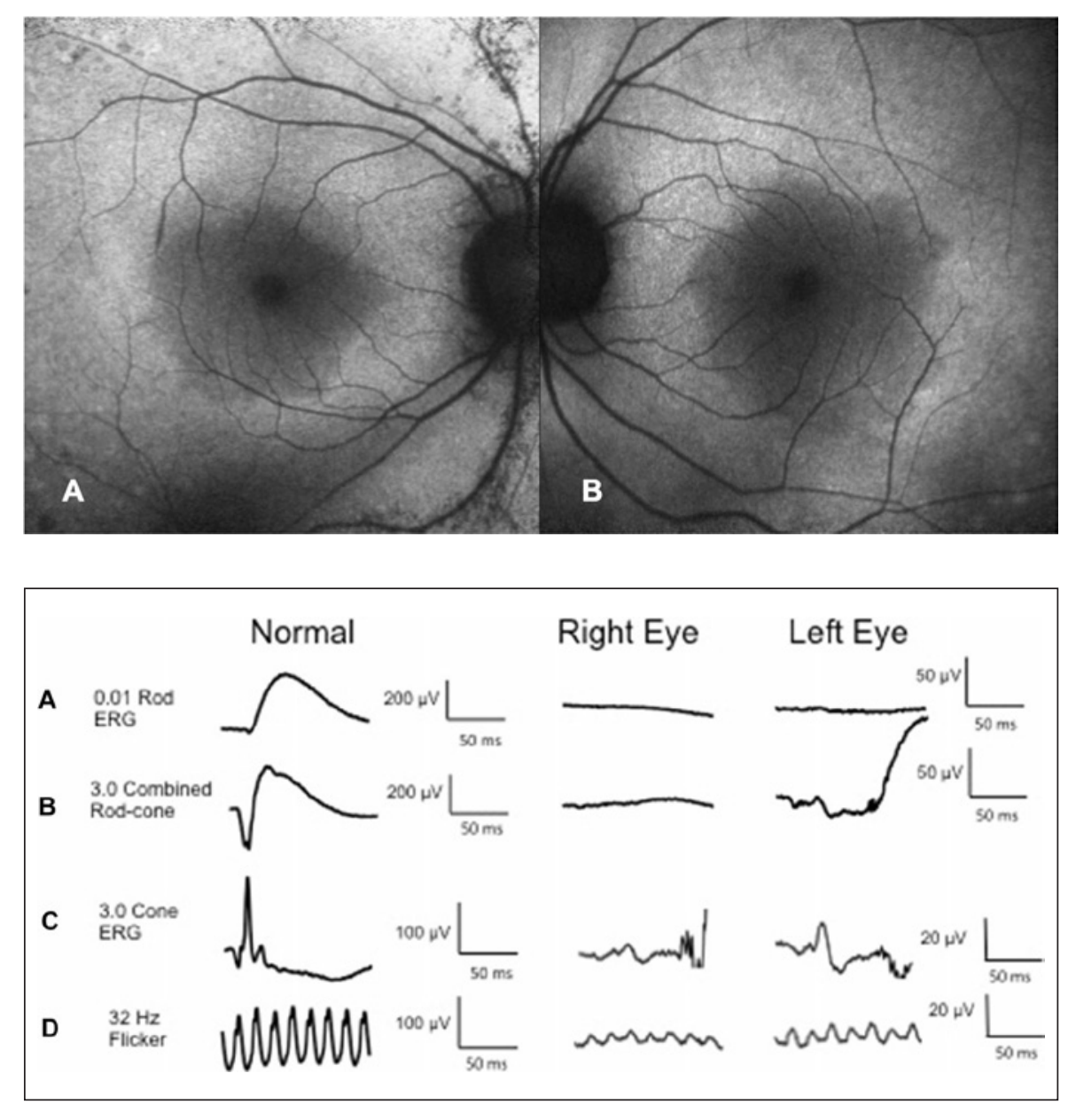

Abb. 6. Die Vollfeld-Elektroretinographie ergab a eine verminderte Stäbchen-isolierte b-Welle im ERG $(0,1)$ beidseitig, konsistent mit einer stark verminderten Stäbchenfunktion; b skotopisches ERG $(3,0)$ mit abgeschwächter kombinierter ZapfenStäbchen-Antwort im RA und $10 \%$ der normalen a- und b-Welle im LA; c Zapfen-Restantwort im ERG $(3,0)$ mit $5 \%$ normaler Amplitude und verzögerter Gipfelzeit beidseitig; $\mathbf{d}$ photopisches Flimmer-ERG $(3,0,32 \mathrm{~Hz})$ vermindert auf $10 \%$ der normalen Amplitude mit verzögerter Gipfelzeit beidseitig. aus durchgeführte Fluoreszenzangiografie (FA) zeigte in beiden Augen eine fehlende periphere Perfusion mit vereinzelten Fluoreszeinleckagen aus Gefäßen und im Bereich der Papille in der Spätphase (Abb. 4 a-b). Die Fundusautofluoreszenz ergab eine diffuse umlaufende Hyperautofluoreszenz im parafovealen Bereich beidseitig (Abb. 5). Der Befund einer nach dem Protokoll der International Society for Clinical Electrophysiology of Vision durchgeführten Vollfeld-Elektroretinographie (ERG) stand im Einklang mit einer fortgeschrittenen, symmetrischen ZapfenStäbchen-Degeneration beidseitig (Abb. 6).

Laboruntersuchungen, u. a. auf antinukleäre Antikörper, antineutrophile zytoplasmatische Antikörper, C-reaktives Protein, Komplementfaktor C3, Komplementfaktor C4, Rheumafaktor, antizyklisches citrulliniertes Peptid, Kryoglobuline, Lupus-Antikoagulans, Angiotensin-Converting-Enzym, Lysozym, EpsteinBarr-Virus-Antikörper, Zytomegalievirus-Antikörper, Bartonella-Antikörper, auf Virushepatitis, HIV, Borrelien-Antikörper (Lyme-Borreliose) und Syphilis-Treponema, ein QuantiferonTest, ein großes Blutbild und ein grundlegendes Stoffwechselprofil waren allesamt ohne Befund. Der HLA-Test auf die Antigene HLA-A29, -B51 und -B27 negativ. Ein antiretinales Autoantikörper-Panel, das über das Augenimmunologie-Labor am Casey Eye Institute in Auftrag gegeben wurde, war positiv für antiretinale Antikörper gegen 30-kDa Carbonanhydrase II-Protein und 46-
kDa Enolase-Protein. Bei der Serumeiweißelektrophorese zeigte sich ein erhöhter M-Gradient von 0,38 g/dl (IgG-Lambda-Leichtketten). Die Patientin wurde an einen Onkologen überwiesen, der bei ihr MGUS diagnostizierte.

Es fand sich keine Netzhautdystrophie in der Familienanamnese, und eine Panelanalyse von 267 Genen für Netzhautdystrophie durch das Ocular Genomics Institute an der Massachusetts Eye and Ear Infirmary ergab keinen eindeutigen Befund. In Anbetracht der relativ schnellen Progression in Verbindung mit Gefäßleckagen bei der FA und dem positiven Ergebnis für antiretinale Antikörper stand das klinische Bild im Einklang mit einer Autoimmun-Retinopathie (AIR). Aufgrund von Bedenken in Bezug auf eine mögliche karzinomassoziierte Retinopathie (CAR) wurden die Krebsvorsorgeuntersuchungen überprüft. Diese umfassten eine unauffällige Mammographie 9 Monate vor Vorstellung der Patientin, einen negativen fäkalen okkulten Bluttest 2 Monate vor Vorstellung, eine Koloskopie ohne maligne Läsionen 5 Jahre vor Vorstellung sowie einen unauffälligen PAP-Abstrich 1 Jahr vor Vorstellung. Eine CT von Thorax- und Abdomen sowie eine MRT des Gehirns und der Orbitae ergaben ebenfalls keine Malignität.

Bei der Patientin wurde eine Behandlung mit Prednison 70 mg (1 $\mathrm{mg} / \mathrm{kg} / \mathrm{Tag}$ ) mit langsamer Dosisreduktion eingeleitet, und im darauf folgenden Monat wurde bei ihr mit der Verabreichung von 
Mycophenolat-Mofetil $1500 \mathrm{mg}$ zweimal täglich begonnen. Es kam bei ihr zu mehreren Episoden schälender Haut, die nach dem Absetzen von Mycophenolat abklangen. Es wurde eine Behandlung mit $15 \mathrm{mg}$ Methotrexat wöchentlich eingeleitet, und die Prednison-Dosis der Patientin wurde über einen Zeitraum von 6 Monaten weiter auf $20 \mathrm{mg}$ täglich gesenkt. Unter diesem Behandlungsschema kam es zu einer Besserung ihres Visus auf 20/20 beidseitig und der Goldmann-Gesichtsfeldmessung (Abb. $1 \mathrm{c}-\mathrm{d}$ ), zu einem Abklingen des Makulaödems in der OCT (Abb. $3 \mathrm{~d}$ ) und einer verminderten Gefäßleckage in der FA (Abb. 4 c-d). Das Ergebnis ihrer Augenhintergrunduntersuchung blieb stabil. Über einen Zeitraum von weiteren 4 Monaten wurde das Prednison komplett ausgeschlichen. Einen Monat später, unter MethotrexatMonotherapie, stellte man bei der Patientin eine fortschreitende Einengung des Gesichtsfeldes im RA fest. Die Methotrexat-Dosis wurde auf 17,5 mg wöchentlich erhöht. Vier Monate später betrug der Visus beidseitig 20/25, und die Patientin bemerkte eine subjektive Verschlechterung des peripheren Sehens im RA. Es wurden eine weitere beidseitige Progression bei der Goldmann-Gesichtsfelduntersuchung (Abb. 1 e-f), ein Wiederauftreten des Makulaödems beidseitig und eine weitere Abschwächung der parafovealen Ellipsoidzone im RA festgestellt. Als Brückentherapie bis zur Gabe von Rituximab-Infusionen erhielt die Patientin in das rechte, ihr schlechteres, Auge eine Einzelinjektion Methylprednisolonacetat unterhalb der Tenon-Kapsel (40 mg). Nach den ersten beiden Rituximab-Infusionen (jeweils $1000 \mathrm{mg}$ ) zeigte sich eine Verbesserung der Einengung in der GVF (Abb. $1 \mathrm{~g}-\mathrm{h}$ ), und das Makulaödem klang in beiden Augen ab. Ihr Visus betrug beidseitig 20/30. Die anschließenden geplanten Infusionstermine konnte die Patientin aufgrund von Verkehrsmittelproblemen nicht wahrnehmen, und als sie 6 Monate nach ihrer letzten Infusion zu uns kam, hatte sich ihr Visus auf 20/40-1 im RA und auf 20/30-1 im LA verschlechtert, ein leichtes zystoides Makulaödem war wieder aufgetreten, und das zentrale Skotom im RA hatte sich vergrößert. Seit kurzem erhält sie wieder Rituximab-Infusionen. Über den klinischen Verlauf hinweg blieb die MGUS stabil.

\section{Diskussion und Schlussfolgerungen}

MGUS ist eine häufige prämaligne Erkrankung der Plasmazellen. Insgesamt 3,2\% der betroffenen Patienten sind über 50 Jahre, 5,3\% über 70 Jahre alt [6]. Die Erkrankung ist gekennzeichnet durch einen Anstieg von monoklonalem Immunglobulin (MProtein) auf unter $30 \mathrm{~g} / \mathrm{l}$, ein Vorkommen klonaler Plasmazellen im Knochenmark von unter 10\% sowie das Fehlen von Folgeschäden, die auf ein Multiples Myelom zurückzuführen sind (Hyperkalzämie, Niereninsuffizienz, Anämie, Knochenläsionen). Bei Patienten mit IgM-MGUS kommt es häufig zu einer Progression zu Morbus Waldenström (MW), während bei Patienten mit IgAoder IgG-MGUS die Erkrankung mit einer Rate von 1\% pro Jahr $\mathrm{zu}$ einem Multiplen Myelom fortschreitet [7]. Unsere Patientin wies eine niedrige M-Protein-Last auf, was ihr Risiko für ein Fortschreiten zu einem Multiplen Myelom verringert. Ungeachtet dessen werden bei ihr weiter regelmäßig onkologische Kontroll- untersuchungen mit serieller Bestimmung des monoklonalen Proteins durchgeführt.

Auch wenn bereits okuläre Manifestationen der MGUS beschrieben wurden [3-5], liegen unseres Wissens bislang keine Berichte über eine MGUS-assoziierte Autoimmun-Retinopathie vor. Die AIR ist gekennzeichnet durch eine Dysfunktion der Photorezeptoren und das Vorliegen antiretinaler Autoantikörper mit damit einher gehenden visuellen Symptomen, u.a. Gesichtsfeldausfällen, einem verminderten Visus, Skotomen, Nachtblindheit und Photopsien. Man unterscheidet bei der AIR einen paraneoplastischen und einen nicht paraneoplastischen Subtyp, wobei der paraneoplastische Subtyp weiter in eine CAR und eine melanomassoziierte Retinopathie (MAR) unterteilt werden kann [8, 9]. Die CAR tritt am häufigsten in Verbindung mit dem kleinzelligen Lungenkarzinom auf, wird jedoch auch im Zusammenhang mit gynäkologischen Krebsarten und Mammakarzinomen, anderen soliden malignen Tumoren und malignen hämatologischen Erkrankungen beobachtet [10,11]. Patienten mit nicht paraneoplastischer AIR (npAIR) sind häufig Frauen im 6. und 7. Lebensjahrzehnt (wie auch unsere Patientin), die oftmals Autoimmunkrankheiten in der familiären oder persönlichen Anamnese aufweisen. Unsere Patientin berichtete von einem nicht näher angegebenen Familienmitglied mit rheumatoider Arthritis. Bei unserer Patientin wurden umfangreiche Untersuchungen durchgeführt, mit negativem Befund für Malignität, aber positivem Befund für MGUS, eine nicht maligne neoplastische Erkrankung.

Während MGUS und Multiples Myelom bislang nicht mit einer Autoimmun-Retinopathie in Zusammenhang gebracht worden sind, liegen Berichte über CAR in Verbindung mit MW vor. Liu et al. beschrieben einen Patienten mit diagnostiziertem MW, vorbehandelt mit therapeutischer Plasmapherese, der Skotome, Photopsien und verschwommenes Sehen entwickelte [12]. Die Untersuchungen ergaben unterdrückte skotopische und photopische Antworten in der Vollfeld-ERG mit Autoantikörpern gegen 35$\mathrm{kDa}$ Glycerinaldehyd-3-phosphat-Dehydrogenase sowie gegen 43- und 44-kDa unspezifizierte Netzhautproteine. Mit der Plasmapherese-Therapie konnte seine CAR erfolgreich stabilisiert werden. Sen et al. beschrieben einen Patienten mit MW, bei dem zunächst eine Zapfen-Dysfunktion vorlag, der aber später Nachtblindheit entwickelte und bei der ERG zusätzlich eine StäbchenDysfunktion zeigte [13]. Immunhistochemisch wurde ein Autoantikörper gegen das angrenzende Cilium an der Grenze zwischen den Innen- und Außensegmenten der Photorezeptoren identifiziert. Die Krankheit schritt deutlich langsamer fort als häufig bei CAR beobachtet. Eine Rituximab-Therapie brachte bei diesem Patienten keine klinische Besserung. In beiden Fällen war bei den Patienten MW diagnostiziert worden, bevor sie visuelle Symptome entwickelten, bei unserer Patientin hingegen wurde die MGUS initial im Rahmen der Abklärung ihrer Retinopathie festgestellt.

Während MW und Multiples Myelom im Gegensatz zu MGUS, einem nicht malignen neoplastischen Krankheitsprozess, als maligne Erkrankungen eingestuft werden, liegt allen drei Krankheitsbildern jedoch ein Mechanismus aus Immundysregulation, 
aberranter Plasmazellproliferation und einer resultierenden Produktion klonaler Antikörper zugrunde. Die Immundysregulation erklärt vermutlich die zwischen MGUS und einer Reihe von Autoimmunerkrankungen, u.a. Spondylitis ankylosans, Polymyositis, rheumatoider Arthritis, Polymyalgia rheumatica, perniziöser Anämie und demyelinisierender Neuropathie, beobachteten Zusammenhänge [14]. Es liegt nahe, dass eine dysregulierte Antikörperproduktion der Entwicklung einer AIR bei Patienten mit MGUS zugrunde liegen könnte, auch wenn wir nicht ausschließen können, dass unsere Patientin AIR unabhängig von der MGUS entwickelte. Verschiedene retinale Antikörper werden mit der AIR in Zusammenhang gebracht, u.a. Autoantikörper gegen Recoverin, die innere plexiforme Schicht, die innere Netzhautschicht, Transducin- $\alpha$ in Stäbchen, $\alpha$-Enolase und Carbonanhydrase II [15]. Die beiden letztgenannten lagen bei unserer Patienten vor.

Eine der Behandlungsoptionen bei AIR sind Immunsuppressiva, die eine Verringerung der zirkulierenden antiretinalen Antikörper bewirken. Zur Behandlung werden u. a. lokale bzw. systemische Kortikosteroide, Cyclosporin, Mycophenolat-Mofetil, Methotrexat, Azathioprin, IVIG und Plasmapherese angewendet [8, $9,16]$. Außerdem liegen Berichte über lang wirksame intravitreale Fluocinolonacetonid-Implantate vor. Sie werden zur Behandlung von Patienten mit paraneoplastischer und nicht paraneoplastischer AIR angewendet, die auf eine systemische Therapie nicht angesprochen oder diese nicht vertragen haben [17]. In der letzten Zeit hat sich Rituximab, ein Immunsuppressivum, das an das Protein CD20 auf B-Lymphozyten bindet, bei einigen Patienten als wirksam zur Stabilisierung oder Besserung der klinischen Symptome und Labormarker von CAR, MAR und npAIR erwiesen $[18,19]$. Dies galt auch für unsere Patientin, bei der es trotz initialer Besserung unter Prednison und Methotrexat später zu einer Progression unter diesen Therapien kam. Nach der Verabreichung von Rituximab-Infusionen verbesserten sich die Ergebnisse von GVF und OCT. Weitere Kontrolluntersuchungen sind erforderlich um zu beurteilen, ob die Patientin eine anhaltende Response auf Rituximab zeigt.

Der vorliegende Bericht zeigt, dass MGUS bei der Differenzialdiagnose von Patienten mit einer Autoimmun-Retinopathie in Betracht gezogen werden sollte. Zudem kann eine okuläre Symptomatik die Erstmanifestation von MGUS darstellen. Dies zu erkennen kann einen früheren Nachweis und eine frühzeitigere Einleitung der Überwachung auf ein Fortschreiten zu MW oder Multiplem Myelom ermöglichen. Augenärzte sollten eine Rituximab-Therapie bei MGUS-assoziierter und anderen Formen von
AIR, die auf andere Behandlungen nicht angesprochen haben, in Betracht ziehen.

\section{Danksagung}

Kari Branham, MS und CGC assistierten bei der genetischen Bewertung und Untersuchung der Patientin.

\section{Autorenbeiträge}

EE: Analyse und Auswertung der klinischen Daten sowie Manuskriptentwurf. GA: Erhebung und Auswertung der klinischen Daten, Überarbeitung des Manuskripts. NK: Erhebung und Auswertung der klinischen Daten, Überarbeitung des Manuskripts. AF: Erhebung, Analyse und Auswertung der klinischen Daten, substanzielle Überarbeitung des Manuskripts. Alle Autoren haben die finale Fassung des Manuskripts gelesen und freigegeben.

\section{Finanzielle Unterstützung}

AF wird unterstützt durch ein K12-Stipendium der National Institutes of Health (K12EY022299).

\section{Verfügbarkeit der Daten und Materialien}

Die Daten dieser Studie sind über die Korrespondenzautorin auf Anfrage erhältlich.

\section{Genehmigung durch die Ethikkommission und Einwilli- gung in die Teilnahme}

Gemäß den institutionellen Leitlinien war für dieses Manuskript keine Genehmigung durch das Institutional Review Board erforderlich. Die Patientin erteilte mündlich und schriftlich ihre Einwilligung in die Teilnahme.

\section{Zustimmung zur Veröffentlichung}

Von der Patientin wurde nach Aufklärung eine schriftliche Einverständniserklärung für die Veröffentlichung der vorliegenden Arbeit und der dazugehörigen Bilder eingeholt. Eine Ausfertigung der schriftlichen Einverständniserklärung kann auf Anfrage eingesehen werden.

\section{Disclosure Statement}

Die Autoren erklären, dass keine Interessenskonflikte bestehen.

\section{Lizenzangabe}

Eton EA, Abrams G, Khan NW, Fahim AT: Autoimmune retinopathy associated with monoclonal gammopathy of undetermined significance: a case report. BMC Ophthalmology 2020;20:153. (DOI: 10.1186/s12886-02001423-y). @ 2020 The Author(s). (Übersetzung; Abkürzungen und Publisher's Note gekürzt), lizensiert unter CC BY 4.0 (https://creativecommons.org/licenses/by/4.0/deed.de).

\section{Literatur}

Die Literatur ist unter www.karger.com/doi/10.1159/000510227 abrufbar. Published online: 18. August 2020 